\title{
Effects of Intravenous Nifekalant as a Lifesaving Drug for Severe Ventricular Tachyarrhythmias Complicating Acute Coronary Syndrome
}

\author{
Satoru Yusu, MD; Takanori Ikeda, MD; Hisaaki Mera, MD; Mutsumi Miyakoshi, MD; \\ Yosuke Miwa, MD; Atsuko Abe, MD; Takehiro Tsukada, MD; \\ Haruhisa Ishiguro, MD; Hisashi Shimizu, MD; Hideaki Yoshino, MD
}

\begin{abstract}
Background: Intravenous amiodarone (AMD) has been used for the treatment of ventricular tachycardia/fibrillation (VT/VF) in emergency care medicine. However, AMD acts slowly and is occasionally accompanied by hypotension and bradycardia. The antiarrhythmic effect of intravenous nifekalant (NIF) was assessed in patients with VT/VF complicating acute coronary syndrome (ACS) according to our study protocol.

Methods and Results: Among a series of 1,143 ACS patients, 41 patients who suffered sustained VT/VF were enrolled; 19 failed to respond to a preceding lidocaine (LID) injection. NIF was given first as an intravenous bolus injection $(0.2 \mathrm{mg} / \mathrm{kg})$ and then as a continuous intravenous infusion at a relatively low dose level $(0.2 \mathrm{mg}$. $\left.\mathrm{kg}^{-1} \cdot \mathrm{h}^{-1}\right)$. Sustained VT/VF was successfully inhibited by NIF in 34 patients $(83 \%)$. In subgroup analysis, NIF achieved VT/VF inhibition in 79\% of patients who received preceding LID and in $86 \%$ of patients who received direct NIF. There were no significant changes in systolic blood pressure or heart rate following NIF therapy. A corrected QT interval was significantly prolonged $(\mathrm{P}<0.01)$, whereas torsade de pointes developed in only 1 patient $(2 \%)$.
\end{abstract}

Conclusions: An intravenous bolus injection and subsequent continuous infusion of NIF at a relatively low dosage were effective in treating severe ventricular tachyarrhythmias complicating ACS, reducing the potential risk of proarrhythmia. (Circ J 2009; 73: 2021-2028)

Key Words: Acute coronary syndrome; Class III antiarrhythmic drug; Emergency care; Nifekalant; Ventricular tachyarrhythmia

$\mathbf{M}$ any centers now perform percutaneous coronary intervention (PCI) for the treatment of acute coronary syndrome (ACS) and, accordingly, the number of patients who have eluded progression of myocardial damage and gained post-ACS conservation of cardiac function has been increasing. ${ }^{1,2}$ Life-threatening ventricular tachyarrhythmias, which occur during the acute phase of ACS, still constitute a major cause of acute-phase death of ACS patients, stressing the importance of their management. ${ }^{3}$

Lidocaine (LID), a class IB antiarrhythmic drug with a modest negative inotropic effect on cardiac function, has mainly been used for the treatment of sustained ventricular tachycardia (VT) or ventricular fibrillation (VF) complicating the acute phase of ACS. However, LID was excluded from the list of drugs to be used because there was no evidence to support its antiarrhythmic effect on sustained $\mathrm{VT} / \mathrm{VF}$, according to the guidelines for "the management of patients with ST-elevation myocardial infarction" 4,5 or "cardiopulmonary resuscitation and emergency cardiovascular care". ${ }^{6}$ Alternatively, intravenous amiodarone (AMD), a class III antiarrhythmic drug, which was demonstrated to be superior in usefulness to placebo in the ARREST Study ${ }^{7}$ and LID in the ALIVE Study, ${ }^{8}$ and was recommended for use as a first-line drug for treatment of VT/VF in patients with ACS. However, it has been reported that the antiarrhythmic effect of AMD is slow to act, ${ }^{9}$ and that its use is occasionally accompanied by adverse drug reactions such as hypotension and bradycardia, ${ }^{10}$ and thus AMD might not be useful as a first-line drug for the suppression of severe ventricular tachyarrhythmias occurring due to ACS.

Nifekalant (NIF) is classified as a class III antiarrhythmic drug along with AMD, and is available for intravenous injection for the treatment of ventricular tachyarrhythmias. NIF was developed in Japan and is being used solely in Japan at present, and is thus not included in the American and European guidelines. Several studies have demonstrated the efficacy of NIF in arresting or controlling sustained VT/VF. ${ }^{11-15}$ NIF is easy to use for the treatment of VT/VF, developing in association with ACS, because of its slight effects on hemodynamic status and the rapid onset of its beneficial effects. Unlike AMD, however, NIF selectively blocks IKr channels and eventually frequently causes QT interval prolongation during sinus rhythm, and might thus give rise to torsade de pointes (TdP) as a proarrhythmic effect. ${ }^{13,16}$ Its use in the treatment of arrhythmias in ACS

Received May 28, 2009; revised manuscript received June 25, 2009; accepted June 26, 2009; released online September 2, 2009

The Second Department of Internal Medicine, Kyorin University School of Medicine, Tokyo, Japan

Mailing address: Takanori Ikeda, MD, Second Department of Internal Medicine, Kyorin University School of Medicine, 6-20-2 Shinkawa, Mitaka, Tokyo 181-8611, Japan. E-mail: iket@ks.kyorin-u.ac.jp

All rights are reserved to the Japanese Circulation Society. For permissions, please e-mail: cj@j-circ.or.jp 


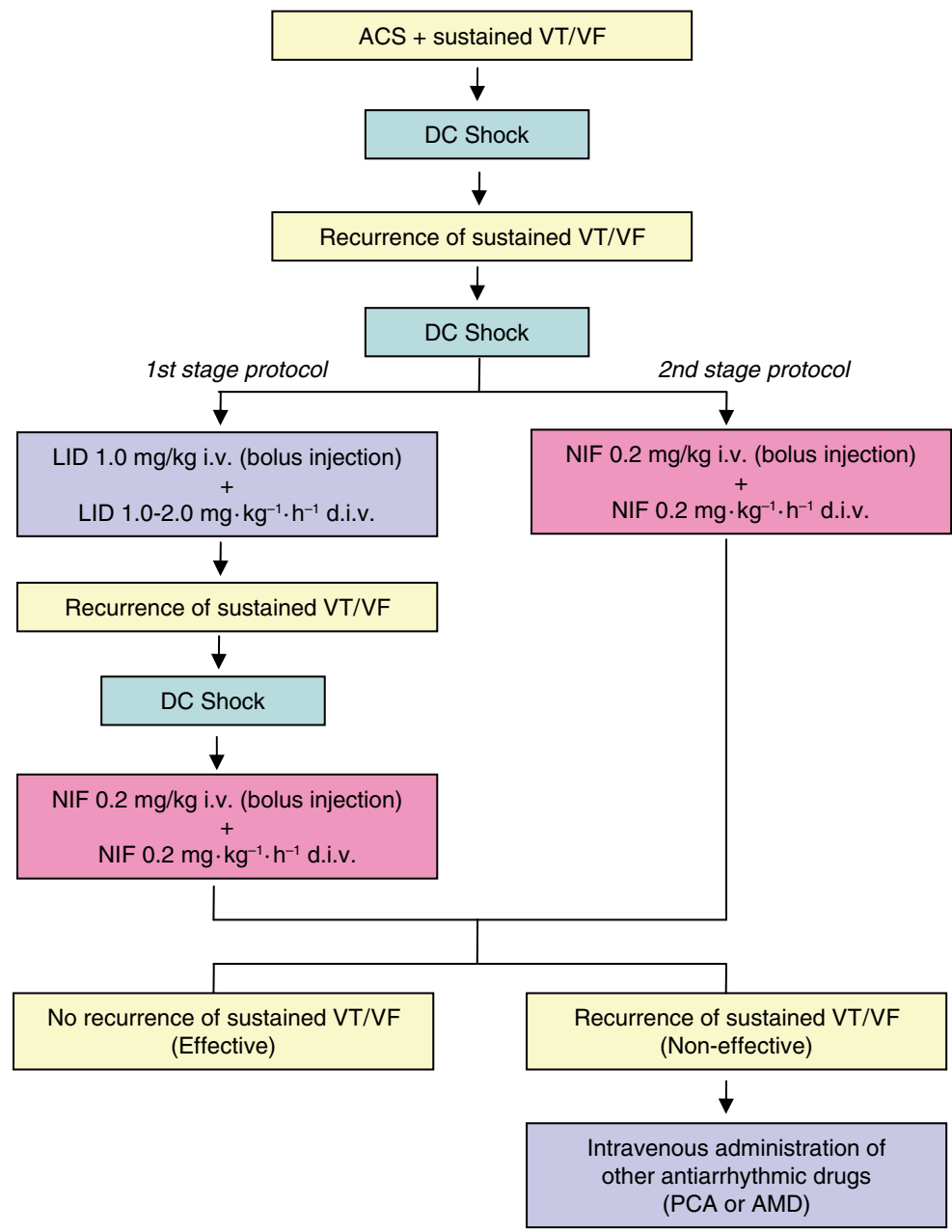

Figure 1. Study protocol. ACS, acute coronary syndrome; DC, direct current; LID, lidocaine; VT, ventricular tachycardia; VF, ventricular fibrillation; LID, lidocaine; NIF, nifekalant; PCA, procainamide; AMD, amiodarone. patients is thus limited.

In the present study, we assessed the efficacy and safety of intravenous NIF with our protocol, that is, intravenous bolus injection followed by continuous intravenous infusion at a relatively low dose level, in the emergency care of patients with severe ventricular tachyarrhythmias complicating ACS.

\section{Methods}

\section{Study Patients}

The study population consisted of 41 patients ( 29 men and 12 women; mean age: $69 \pm 14$ years) who suffered sustained VT/VF producing circulatory failure and experienced recurrence even after direct-current (DC) shock, from among a series of 1,143 patients admitted for emergency treatment because of diagnosed ACS to the Coronary Care Unit of Kyorin University Hospital during the 7-year period from January 2002 to December 2008. For each patient, written or verbal informed consent was obtained prior to entry from the patient or from his/her family member.

The diagnosis of ACS was based on chest-related symptoms and a 12-lead electrocardiogram. Acute myocardial infarction (AMI) was diagnosed if there was elevation of serum myocardial enzymes, or as unstable angina pectoris (UAP) if there was no such elevation. Treatment undertaken prior to initiation of antiarrhythmic drug therapy was according to the guidelines for treatment of ACS.4,5,17 In
ACS patients whose condition was judged to require revasucularization therapy during the acute phase, such as PCI or coronary artery bypass graft (CABG) surgery, the above therapeutic measure was performed without delay. The criteria for exclusion of subjects from this study included the presence of cardiac arrest on arrival at this hospital, patients administered any other antiarrhythmic drugs before transferral to our hospital, the presence of renal failure (serum creatinine $>2.0 \mathrm{mg} / \mathrm{dl}$ ), or the presence of sustained VT not adversely affecting hemodynamics. Patients meeting any of these criteria were excluded from this study.

The present study was conducted with the approval of the Ethics Committee on Medical Research of Kyorin University Hospital.

\section{Study Protocol}

The protocol for the present study is shown in Figure 1. If life-threatening VT/VF occurred after the diagnosis of ACS had been established, a DC shock (200-360 joules for a monophasic defibrillator, or 100-150 joules for a biphasic defibrillator) was initially performed to promptly terminate the arrhythmia. If the arrhythmic event subsequently recurred, the condition was judged to be an arrhythmia requiring intravenous antiarrhythmic drug therapy, and NIF was administered according to either of the following 2 protocols (1st stage or 2 nd stage). 
Table 1. Study Population With DC Shock-Resistant Sustained VT/VF Complicating ACS

\begin{tabular}{lccc}
\hline & All patients & $\begin{array}{c}1 \text { st stage } \\
\text { protocol patients }\end{array}$ & $\begin{array}{c}\text { 2nd stage } \\
\text { protocol patients }\end{array}$ \\
\hline Number of patients & 41 & 19 & 22 \\
Age (years) & $69 \pm 14$ & $70 \pm 17$ & $68 \pm 11$ \\
M/F (n) & $29 / 12$ & $11 / 8$ & $18 / 4$ \\
Documented ventricular tachyarrhythmias & & & \\
Sustained VT & $27(66 \%)$ & $12(63 \%)$ & $15(68 \%)$ \\
VF & $14(34 \%)$ & $7(37 \%)$ & $7(32 \%)$ \\
Etiology of ACS & $31(76 \%)$ & $14(74 \%)$ & $17(77 \%)$ \\
AMI (n) & $10(24 \%)$ & $5(26 \%)$ & $5(23 \%)$ \\
UAP (n) & $23(74 \%)$ & $11(79 \%)$ & $12(71 \%)$ \\
Myocardial injury area & $8(26 \%)$ & $3(21 \%)$ & $5(29 \%)$ \\
Left anterior wall (n) & $0(0 \%)$ & $0(0 \%)$ & $0(0 \%)$ \\
Left inferior wall (n) & & & \\
Left lateral wall (n) & $22(54 \%)$ & $8(42 \%)$ & $14(64 \%)$ \\
Revascularization procedures & $8(20 \%)$ & $5(26 \%)$ & $3(14 \%)$ \\
PCI (n) & $43 \pm 12$ & $38 \pm 11$ & $49 \pm 11$ \\
CABG surgery (n) & $31(76 \%)$ & $14(74 \%)$ & $17(77 \%)$ \\
LVEF (\%) & & \\
Use of catecholaminergic drugs & & & \\
\hline
\end{tabular}

DC, direct current; VT, ventricular tachycardia; VF, ventricular fibrillation; ACS, acute coronary syndrome; AMI, acute myocardial infarction; UAP, unstable angina pectris; PCI, percutaneous coronary intervention; CABG, coronary artery bypass graft; LVEF, left ventricular ejection fraction.

\section{1st Stage Protocol}

This protocol was followed during the 3-year period from January 2002 to December 2004. Prior to administration of $\mathrm{NIF}$, a $1.0-\mathrm{mg} / \mathrm{kg}$ bolus dose of LID was injected intravenously in accordance with the conventional treatment scheme for concurrent ventricular tachyarrhythmia in patients with ACS. ${ }^{17}$ This was followed by a continuous intravenous infusion of LID at $1.0-2.0 \mathrm{mg} \cdot \mathrm{kg}^{-1} \cdot \mathrm{h}^{-1}$. If VT/VF causing circulatory failure subsequently recurred, the condition was judged to be arrhythmia refractory to LID, and NIF was then administered using the following procedure. The arrhythmia was terminated by DC shock, and then a $0.2-\mathrm{mg} / \mathrm{kg}$ bolus dose of NIF [1 vial $(50 \mathrm{mg})$ dissolved in $20 \mathrm{ml}$ of $5 \%$ glucose fluid] was immediately given intravenously, and maintenance intravenous infusion was administered thereafter at $0.2 \mathrm{mg} \cdot \mathrm{kg}^{-1} \cdot \mathrm{h}^{-1}$. No increase in NIF dosage was performed in this study to avoid the occurrence of $\mathrm{TdP}$ due to $\mathrm{QT}$ interval prolongation. In patients with sustained VT/VF that recurred even after continuous intravenous infusion of NIF, the arrhythmia was terminated with the same procedure and then intravenous administration of another antiarrhythmic drug (procainamide: PCA) was undertaken in place of NIF.

\section{2nd Stage Protocol}

This protocol was followed during the 4-year period from January 2005 to December 2008. Without preceding LID administration, NIF was immediately administered with an intravenous bolus dose of $0.2 \mathrm{mg} / \mathrm{kg}$, followed by a maintenance intravenous drip infusion at $0.2 \mathrm{mg} \cdot \mathrm{kg}^{-1} \cdot \mathrm{h}^{-1}$. If sustained VT/VF recurred, the arrhythmia was terminated by a DC shock, as in the stage 1 protocol, and then intravenous administration of another antiarrhythmic drug (PCA during the period up to June 2007, or AMD from July 2007 onwards) was performed without an increase in dosage of NIF.

\section{Assessment of Clinical Background}

Assessment variables in terms of clinical features used in the study included age, gender, type of ventricular tachyar- rhythmia, type of ACS, details of myocardiopathic region, whether any revascularization therapy was performed and details of that therapy if administered, mean left ventricular ejection fraction (LVEF), serum $\mathrm{K}^{+}$level, and serum creatinine level. Ventricular tachyarrhythmia was described in terms of the condition just prior to initiation of NIF therapy. All ventricular tachyarrhythmias subjected to evaluation were persistent in nature. Ventricular tachyarrhythmias were defined here as VT if the QRS configuration in the ECG was clearly discernible, and otherwise as VF.

\section{Assessment of Effects of NIF Therapy}

Therapeutic responses to intravenous NIF medication were evaluated with respect to arrhythmia-preventive effects, changes in systolic blood pressure, changes in heart rate during sinus rhythm, changes in corrected QT (QTc) inter$\mathrm{val}$, and emergence of proarrhythmic effects as an adverse drug reaction, that is, the appearance of TdP, compared with the status before NIF therapy. An antiarrhythmic effect was considered evident if sustained VT/VF completely disappeared following administration of NIF. Whether there were any differences in therapeutic response to NIF between the 2 study protocols (1st stage protocol vs 2nd stage protocol) was also assessed.

In this study, assessments were also made regarding survival based on the outcome over a 3-month follow up in the cases studied.

\section{Statistical Analysis}

All analyses were carried out using statistical software (SPSS version 15.0 for Windows). Non-discrete data were expressed as the mean \pm standard deviation. Comparisons of assessment variables between patients in the 1st stage protocol and those in the 2nd stage protocol, between responders and non-responders to NIF therapy, and between pre- and post-NIF therapy were performed using the unpaired Student's t-test. Comparative tests were all 2-sided, and differences found were considered statistically significant if $\mathrm{P}<0.05$. 


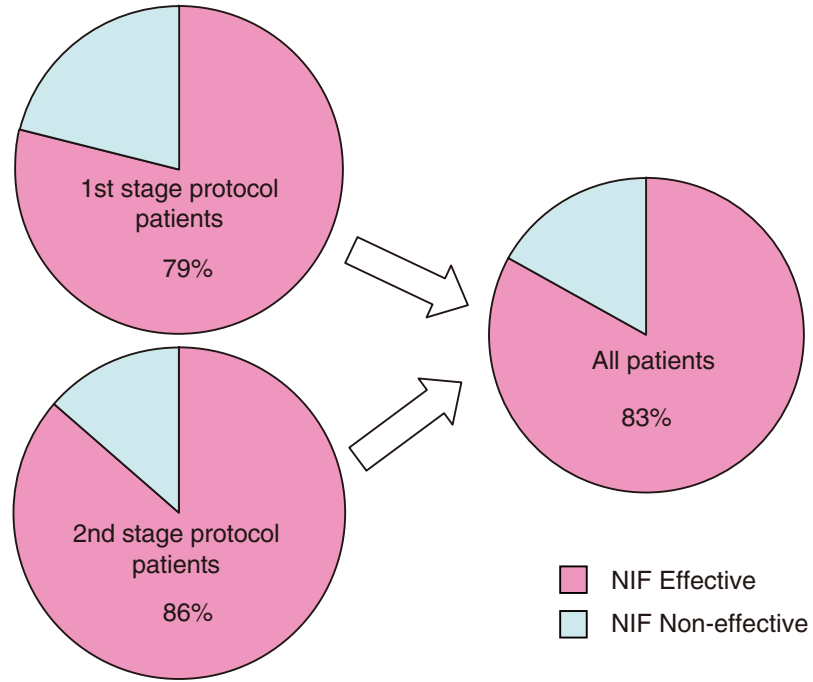

Figure 2. Antiarrhythmic effect of intravenous nifekalant (NIF) infusion.

\section{Results}

\section{Clinical Characteristics}

The clinical characteristics of the study patients are summarized in Table 1. The mean age was $69 \pm 14$ years, and there were 29 male patients. The type of ventricular tachyarrhythmia subjected to evaluation of response to NIF therapy was VT in 27 patients and VF in 14 patients. The type of ACS was AMI in 31 patients and UAP in 10 patients. The myocardopathic region was the anterior wall in 23 patients, the inferior wall in 8 patients, and the lateral wall in none. The mean peak creatinine kinase level in the patients with AMI was $4,487 \pm 3,611 \mathrm{IU} / \mathrm{dl}$. Thirty of the 41 patients (73\%) underwent revascularization therapy prior to initiation of NIF therapy, $22(73 \%)$ of whom underwent PCI (AMI: 21 patients, UAP: 1 patient). There were 8 patients (27\%) in whom CABG was performed (AMI: 5 patients, UAP: 3 patients). In the remaining 11 patients (26\%), no revascu- larization therapy was performed prior to initiation of NIF therapy for reasons such as severe inflammatory reactions due to concurrent pneumonia or other infection, diagnosis of vasospastic angina, resolution of myocardial ischemia by drug medication, and advanced age. The mean LVEF before antiarrhythmic drug treatment was $43 \pm 12 \%$. There were no notable differences in clinical characteristics between patients in the 1st stage protocol and those in the 2nd stage protocol.

All patients with AMI in this study had received intravenous drip infusion of catecholaminergic drugs such as dobutamine and dopamine before treatment with NIF.

\section{Effects of NIF Therapy With This Study Protocol}

1. Antiarrhythmic Effect In 34 of the 41 patients (83\%) studied, ventricular tachyarrhythmia was successfully controlled by continuous intravenous infusion of NIF at relatively low-dose levels given directly following the intravenous bolus injection of NIF (Figure 2). In the other 7 patients $(17 \%)$, the attempt to control sustained VT/VF failed, and VT/VF proved refractory to other antiarrhythmic agents (PCA and AMD) and this led to death from arrhythmia. A comparison of clinical features between responders and non-responders to NIF therapy revealed no significant differences (Table 2). In subgroup analysis, an antiarrhythmic effect was evident in 15 of 19 patients (79\%) in the 1st stage protocol (with preceding LID treatment) and in 19 of 22 patients (86\%) in the 2nd stage protocol (direct initiation of NIF treatment). The arrhythmia-preventive effect was thus greater with the 2nd stage protocol than with the 1st stage protocol, although not to a statistically significant extent. As soon as the patient was judged responsive to NIF infusion, oral AMD at $400 \mathrm{mg} /$ day was administered concomitantly for 2-3 days with a gradual reduction of NIF dose to discontinuation. The mean duration of NIF therapy in this study was $3.7 \pm 1.5$ days, and there was no case in which NIF therapy was continued for $\geq 7$ days.

Three-month follow-up data revealed the following prognoses: 26 of the 34 patients $(76 \%)$ who have exhibited an arrhythmia-preventive effect were discharged alive (ie, $63 \%$ of the 41 patients studied). The remaining 8 patients

Table 2. Comparison of Clinical Characteristics Between Patients for Whom NIF Was or Was Not Effective

\begin{tabular}{|c|c|c|c|}
\hline & \multicolumn{2}{|c|}{ NIF } & \multirow{2}{*}{$P$ value } \\
\hline & Effective for patients & Non-effective for patients & \\
\hline Number of patients & 34 & 7 & \\
\hline Age (years) & $68 \pm 14$ & $72 \pm 11$ & NS \\
\hline $\mathrm{M} / \mathrm{F}(\mathrm{n})$ & $23 / 11$ & $6 / 1$ & NS \\
\hline \multicolumn{4}{|c|}{ Documented ventricular tachyarrhythmias } \\
\hline Sustained VT & $23(68 \%)$ & $4(57 \%)$ & NS \\
\hline $\mathrm{VF}$ & $11(32 \%)$ & $3(43 \%)$ & NS \\
\hline \multicolumn{4}{|l|}{ Etiology of ACS } \\
\hline AMI (n) & $26(76 \%)$ & $5(71 \%)$ & NS \\
\hline UAP (n) & $8(24 \%)$ & $2(29 \%)$ & NS \\
\hline \multicolumn{4}{|l|}{ Myocardial injury area } \\
\hline Left anterior wall (n) & $19(56 \%)$ & $4(57 \%)$ & NS \\
\hline Left inferior wall (n) & $7(21 \%)$ & $1(14 \%)$ & NS \\
\hline Left lateral wall (n) & $0(0 \%)$ & $0(0 \%)$ & NS \\
\hline \multicolumn{4}{|l|}{ Revascularization procedures } \\
\hline PCI (n) & $21(62 \%)$ & $1(14 \%)$ & NS \\
\hline CABG surgery (n) & $5(15 \%)$ & $3(43 \%)$ & NS \\
\hline $\operatorname{LVEF}(\%)$ & $43 \pm 13$ & $42 \pm 6$ & NS \\
\hline Serum K+ level (mmol/L) & $3.9 \pm 0.5$ & $3.5 \pm 0.6$ & NS \\
\hline Serum creatinin level (mg/dl) & $1.2 \pm 0.3$ & $1.3 \pm 0.6$ & NS \\
\hline
\end{tabular}

NIF, nifekalant. Other abbreviations see in Table 1. 


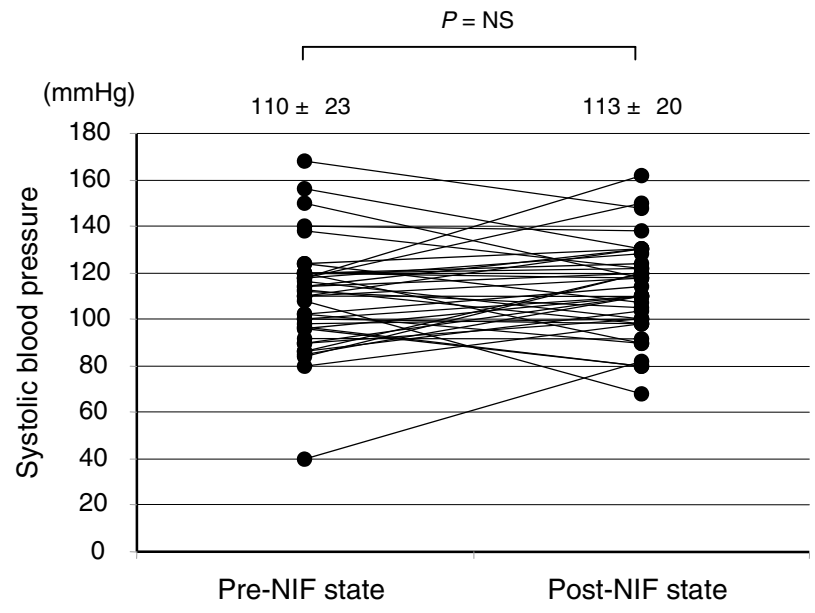

Figure 3. Comparison of systolic blood pressure during sinus rhythm between the pre- and post-state of nifekalant (NIF).

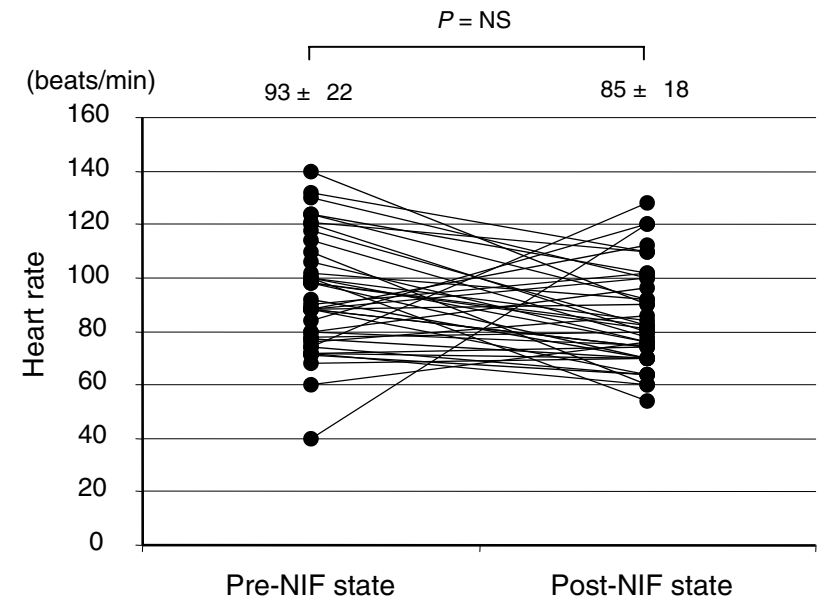

Figure 4. Comparison of heart rate during sinus rhythm between the pre- and post-state of nifekalant (NIF).
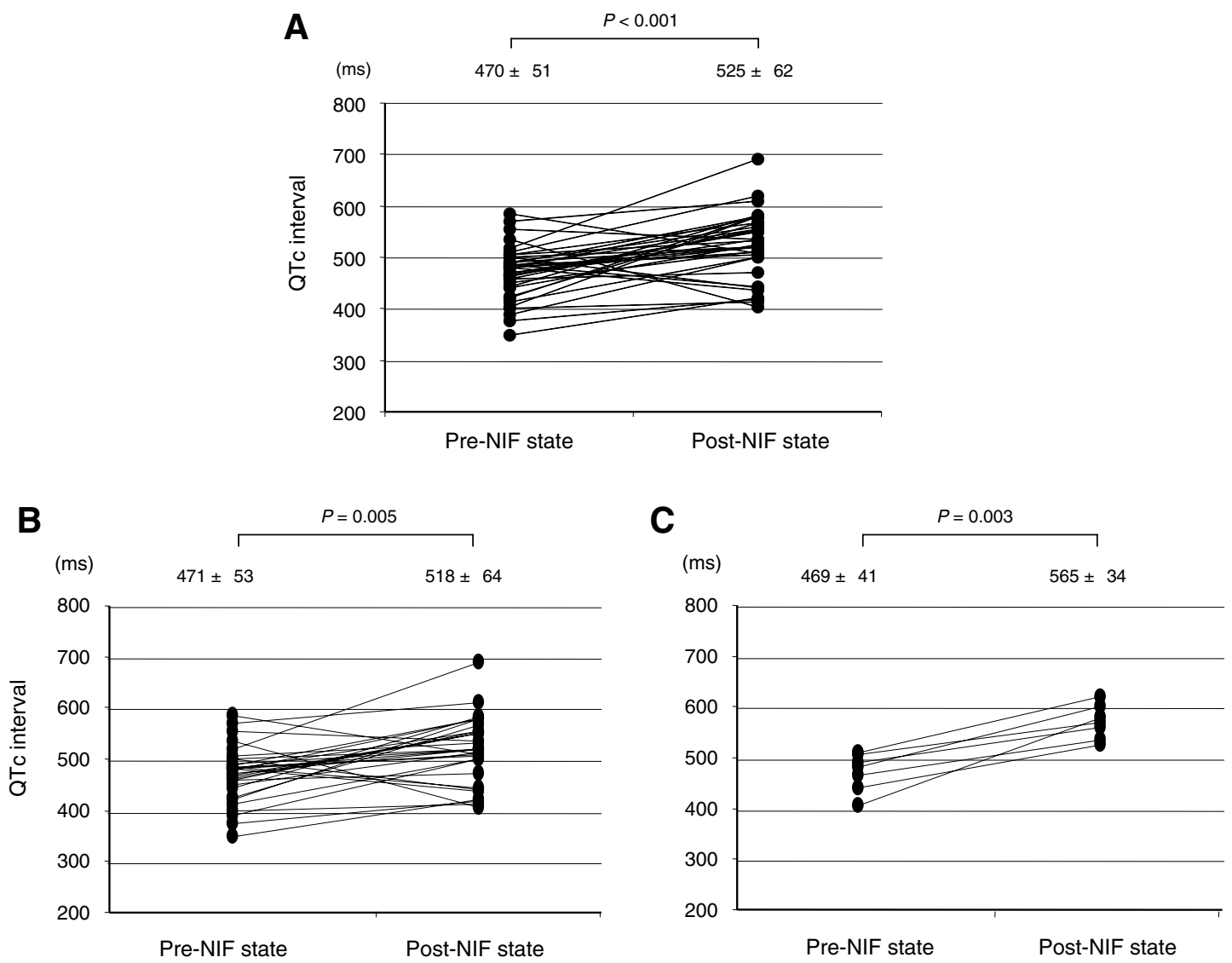

Figure 5. Comparison of the QTc interval during sinus rhythm between the pre- and post-state of nifekalant (NIF). (A) All patients. (B) Responders to NIF therapy. (C) Non-responders to NIF therapy.

escaped death from arrhythmia but eventually died due to heart failure or complications such as severe infection.

2. Effects on Blood Pressure and Heart Rate The mean systolic blood pressure during sinus rhythm after administration of NIF $(113 \pm 20 \mathrm{mmHg})$ did not differ from that before administration of NIF $(110 \pm 23 \mathrm{mmHg})$; blood pressure was thus not affected by NIF (Figure 3). This parameter also did not differ by protocol, that is, the comparison between the 1st stage protocol $(101 \pm 25 \mathrm{mmHg}$ vs
$113 \pm 18 \mathrm{mmHg})$ and the 2 nd stage protocol $(117 \pm 18 \mathrm{mmHg}$ vs $111 \pm 21 \mathrm{mmHg}$ ).

Regarding heart rate during sinus rhythm, a tendency toward decrease was observed after administration of NIF, that is, $93 \pm 22$ beats/min before administration of NIF and $85 \pm 18$ betas/min after administration of NIF, although no significant difference was noted (Figure 4). A comparison by protocol revealed a significant difference for the 1st stage protocol $(100 \pm 25$ beats/min vs $85 \pm 17$ beats/min, $\mathrm{P}=0.04)$ but 


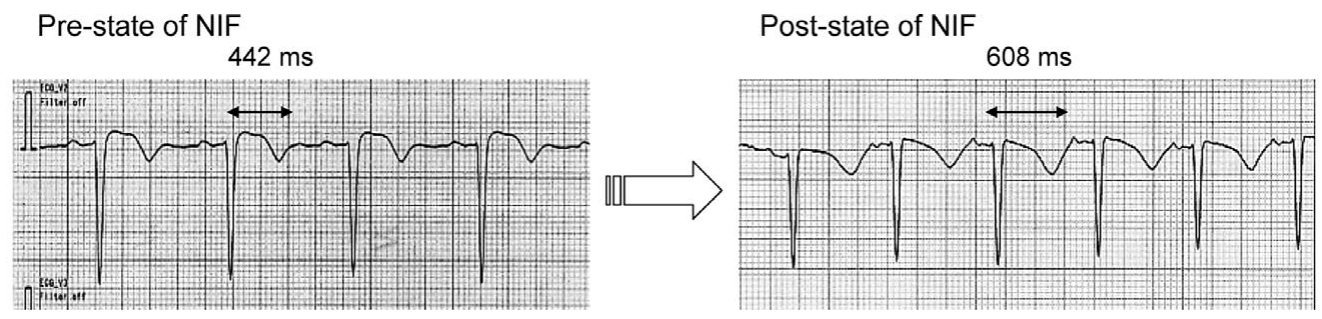

Occurrence of TdP

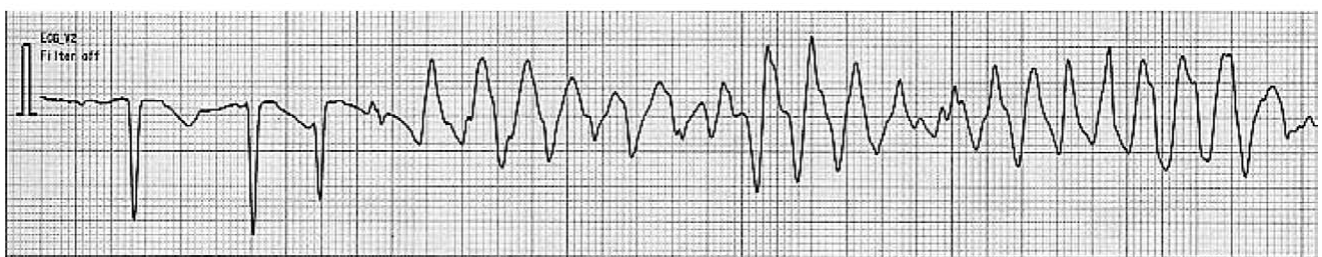

Figure 6. Electrocardiograms in a patient in whom torsade de pointes (TdP) suddenly occurred during the intravenous drip infusion of nifekalant (NIF).

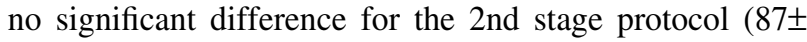
17 beats $/ \mathrm{min}$ vs $84 \pm 19$ beats $/ \mathrm{min}$ ). These findings indicated that preceding LID treatment tended to give rise to bradycardia during NIF therapy.

3. Changes in QT Interval and Proarrhythmic Effects The QTc interval during sinus rhythm had already been prolonged $(470 \pm 51 \mathrm{~ms})$ before administration of NIF due to myocardial ischemia. After administration of NIF, it was further prolonged to $525 \pm 62 \mathrm{~ms}$, with significant prolongation of the QTc interval compared with before administration of NIF $(\mathrm{P}<0.001)$ (Figure 5A). A comparison of QTc prolongation between responders and non-responders to NIF therapy revealed no difference, that is, both groups had significant values (Figure 5B,C). Results were comparable for the 1st stage protocol $(469 \pm 52 \mathrm{~ms}$ vs $544 \pm 59 \mathrm{~ms}, \mathrm{P}<$ $0.01)$ and 2 nd stage protocol $(471 \pm 50 \mathrm{~ms}$ vs $508 \pm 63 \mathrm{~ms}, \mathrm{P}=$ 0.04 ), indicating that this parameter was significantly prolonged regardless of whether preceding LID treatment had been administered.

TdP was observed as a proarrhythmic effect in only 1 of the 41 patients (2\%) receiving NIF in this study (Figure 6). This event occurred on Day 5 of NIF therapy. The patient was with AMI and had received preceding LID treatment and their QTc interval was $442 \mathrm{~ms}$ prior to initiation of NIF therapy. After the start of NIF therapy, their QTc interval increased to $608 \mathrm{~ms}$ and TdP developed. However, the TdP was transient and disappeared promptly following discontinuation of NIF infusion.

\section{Discussion}

Life-threatening ventricular tachyarrhythmias such as VT or VF are likely to develop during the acute phase of ACS, and the occurrence of these arrhythmias has important effects on the prognosis of ACS patients. ${ }^{3}$ Currently, AMD plays a central role in the treatment of arrhythmias in patients with ACS in the USA and Europe. ${ }^{4}$ AMD is a class III antiarrhythmic drug, which exerts a potent antiarrhythmic effect via its ability to prolong the refractory period mainly through a K channel-blocking effect. However, AMD does not have a prompt onset of effect; it has been shown that its onset of action is $6-8 \mathrm{~h}$ after administration, on average. ${ }^{9}$ Because AMD sometimes causes hypotension and brady- cardia, it might not be easy to use as rescue medication.

In Japan, NIF, which is classified as a class III antiarrhythmic drug, as is AMD, is available for clinical use, although its pharmacological effects differ from those of AMD. Use of NIF for the treatment of ventricular tachyarrhythmias has been more common in Japan, because intravenous AMD was not shown in the National Health Insurance reimbursement price list until June 2007. The frequency with which NIF is prescribed for the control of ventricular tachyarrhythmias that occur during the acute phase of ACS is still low. The reasons for this include liability to development of QT interval prolongation and occurrence of TdP during sinus rhythm rather than during tachycardia, owing to its reverse use-dependent blocking effect. ${ }^{18}$ Nevertheless, unlike AMD, NIF has a prompt onset of effect with little influence on hemodynamic status and, pharmacologically, has a much greater inhibitory effect than LID on re-entry, the mechanism of sustained arrhythmias. ${ }^{19}$ NIF might thus become a drug of first choice for the control of sustained VT/VF associated with ACS if an appropriate method of administration, with emphasis laid on safety, can be established.

Ohashi et al ${ }^{14}$ evaluated the VT/VF-controlling effect of continuous intravenous infusion of NIF in 16 patients with ACS and 14 patients with chronic structural heart disease. Treatment was successful in controlling VT/VF in 12 of the 16 patients (75\%) with ACS, and none of these patients experienced worsening of their hemodynamic status. The mean dose level of NIF in their study was $0.19 \pm 0.14 \mathrm{mg}$. $\mathrm{kg}^{-1} \cdot \mathrm{h}^{-1}$, yet the dose ranged up to a maximum of $0.6 \mathrm{mg}$. $\mathrm{kg}^{-1} \cdot \mathrm{h}^{-1}$. The incidence of TdP after administration of NIF occurred in as many as 5 of the 30 patients (17\%). In a study reported by Yoshioka et al, ${ }^{12}$ the effects of electrical defibrillation with DC shock following intravenous NIF administration with or without preceding LID treatment were assessed in patients with cardiopulmonary arrest that developed in association with VT/VF in or out of the hospital. Their study demonstrated that the defibrillatory effect of NIF was decreased when treatment was preceded by LID injection (the defibrillation rate in the patient group given preceding LID treatment was $24 \%$, compared to $81 \%$ in the group directly administered NIF). The above group of investigators ${ }^{12}$ used a protocol, which required admin- 
istration of NIF at a dose of $0.15 \mathrm{mg} / \mathrm{kg}$ by intravenous injection over $5 \mathrm{~min}$ (an additional equal dose was allowed if the arrhythmia was not terminated). In their study, the incidence of $\mathrm{TdP}$ as a proarrhythmic effect was only $5 \%$ ( 1 of 21 patients), in part, because of the low dose used.

In the present study, NIF was initially administered as bolus dose of $0.2 \mathrm{mg} / \mathrm{kg}$ intravenously, similar to the intravenous administration of LID, yet no hemodynamic exacerbation occurred in any of the patients. NIF can be administered by rapid intravenous injection as well. Furthermore, the arrhythmia-preventive effect of NIF was high at $83 \%$ and the incidence of TdP was low at $2 \%$ when intravenous NIF infusion was maintained with a relatively low dose level of $0.2 \mathrm{mg} \cdot \mathrm{kg}^{-1} \cdot \mathrm{h}^{-1}$. Thus, TdP can be avoided as an adverse drug reaction and a noticeable antiarrhythmic effect can be obtained with NIF therapy if administration is performed according to a protocol requiring an intravenous bolus dose followed by maintenance of intravenous NIF infusion at a low dose level. In addition, it appears that NIF might be used as a drug of first choice in ACS patients with high risk of ventricular tachyarrhythmias. Furthermore, a strategy in which NIF is used if the patient has failed to respond to LID does not appear to be appropriate because, as shown in this study, preceding LID treatment is likely to give rise to bradycardia. A strategy in which, if NIF has failed to sufficiently control sustained VT/VF, it is replaced by AMD in accordance with the current guidelines for treatment of ACS, ${ }^{4}$ without continuing NIF at increased doses, appears to be best clinically.

The advantage of NIF use in the emergency care of ACS patients lies in the fact that effective control of ventricular tachyarrhythmias can be expected with a prompt onset of effect without alteration of hemodynamic status variables such as blood pressure. It is difficult to use a drug that takes time until the onset of its effect, ${ }^{16}$ such as AMD, in the emergency care of patients suffering frequent episodes of VT/VF, namely, an electrical storm. Drugs with pharmacological effects like those of NIF are easier to use. In fact, it has been demonstrated by Washizuka et al ${ }^{11}$ that treatment with NIF resulted in the disappearance of the electrical storm in all 6 patients who suffered it, when it was accompanied by deep sedation and refractory to $\beta$-blockers.

Regarding QT interval prolongation during NIF therapy, patients with ACS often already exhibit prolongation of the QT interval as a result of subendocardial ischemia;20,21 it thus seems very likely that the QT interval will be prolonged to a greater extent than expected, leading to development of $\mathrm{TdP}$, if NIF is used at the usual dose level $\left(0.4 \mathrm{mg} \cdot \mathrm{kg}^{-1}\right.$. $\mathrm{h}^{-1}$ ) in such patients. As TdP only rarely occurred during NIF therapy at the dose level used in this study $(0.2 \mathrm{mg}$. $\mathrm{kg}^{-1} \cdot \mathrm{h}^{-1}$ ), intravenous maintenance infusion at this dose level can be recommended for the treatment of ACS patients. Furthermore, it appears advisable to limit the duration of NIF treatment to several days in order to reduce the occurrence of adverse drug reactions (TdP occurred on day 5 of NIF therapy in this study). For this purpose, we recommend that a concomitant oral class III antiarrhythmic drug such as AMD be initiated soon after NIF has proven effective, and that NIF be withdrawn with a gradual reduction of dosage over 2-3 days.

\section{Conclusions}

This is the first study to show the lifesaving effect and safety of antiarrhythmic NIF treatment performed to control severe and sustained VT/VF complicating ACS. NIF can be used as an intravenous bolus injection, and was demonstrated to yield an antiarrhythmic effect in as many as $83 \%$ of patients treated. It was also found that occurrence of TdP due to the development of QT interval prolongation was quite rare when NIF was administered by continuous intravenous infusion at a relatively low dose level for a brief duration. NIF can be recommended as a drug of first choice for the treatment of life-threatening ventricular tachyarrhythmia caused by ACS.

\section{Acknowledgments}

This study was supported, in part, by a Grant-in-Aid (18300157) for Scientific Research from the Ministry of Education, Culture, Sports, Science and Technology of Japan, by a grant from the Fukuda Memorial Foundation for Medical Research (Dr Ikeda), and by a grant from Kyorin University School of Medicine (Dr Ikeda).

\section{References}

1. de Winter RJ, Windhausen F, Cornel JH, Dunselman PH, Janus CL, Bendermacher PE, et al; Invasive versus Conservative Treatment in Unstable Coronary Syndromes (ICTUS) Investigators. Early invasive versus selectively invasive management for acute coronary syndromes. N Engl J Med 2005; 353: 1095-1104.

2. Hirsch A, Windhausen F, Tijssen JG, Verheugt FW, Cornel JH, de Winter RJ, et al. Long-term outcome after an early invasive versus selective invasive treatment strategy in patients with non-ST-elevation acute coronary syndrome and elevated cardiac troponin $\mathrm{T}$ (the ICTUS trial): A follow-up study. Lancet 2007; 369: 827-835.

3. Goldberg RJ, Yarzebski J, Spencer FA, Zevallos JC, Lessard D, Gore JM. Thirty-year trends (1975-2005) in the magnitude, patient characteristics, and hospital outcomes of patients with acute myocardial infarction complicated by ventricular fibrillation. Am J Cardiol 2008; 102: 1595-1601.

4. ACC/AHA 2004 guidelines for the management of patients with STelevation myocardial infarction-Executive summary: A report of the American College of Cardiology/American Heart Association task force on practice guidelines. Circulation 2004; 110: 588-636.

5. JCS 2008 guidelines for the treatment of patients with ST-elevation acute myocardial infarction: A report of the Japanese Circulation Society committee for diagnosis and treatment of cardiovascular diseases. Circ J 2008; 72(Suppl IV): 1347-1442.

6. AHA 2005 guidelines for cardiopulmonary resuscitation and emergency cardiovascular care: ECC committee, subcommittees and task forces of the American Heart Association. Circulation 2005; 112(Suppl IV): IV-1 - IV-203.

7. Kudenchuk PJ, Cobb LA, Copass MK, Cummins RO, Doherty AM, Fahrenbruch CE, et al. Amiodarone for resuscitation after out-ofhospital cardiac arrest due to ventricular fibrillation. $N$ Engl J Med 1999; 341: $871-878$.

8. Dorian P, Cass D, Schwartz B, Cooper R, Gelaznikas R, Barr A, et al. Amiodarone as compared with lidocaine for shock-resistant ventricular fibrillation. $N$ Engl J Med 2002; 346: 884-890.

9. Kato T, Ogawa S, Yamaguchi I, Kasanuki H, Hayakawa K. Efficacy and safety of intravenous amiodarone infusion in Japanese patients with hemodynamically compromised ventricular tachycardia of ventricular fibrillation. J Arrhythmia 2007; 23: 131-139.

10. Desai AD, Chun S, Sung RJ. The role of intravenous amiodarone in the management of cardiac arrhythmias. Ann Intern Med 1997; 127: 294-303.

11. Washizuka T, Chinushi M, Watanabe H, Hosaka Y, Komura S, Sugiura H, et al. Nifekalant hydrochloride suppresses severe electrical storm in patients with malignant ventricular tachyarrhythmias. Circ J 2005; 69: 1508-1513.

12. Yoshioka K, Amino M, Morita S, Nakagawa Y, Usui K, Sugimoto A, et al. Can nifekalant hydrochloride be used as a first-line drug for cardiopulmonary arrest (CPA)?: Comparative study of out-of-hospital CPA with acidosis and in-hospital CPA without acidosis. Circ $J$ 2006; 70: 21-27.

13. Amino M, Yoshioka K, Morita S, Otsuka H, Yamagiwa T, Umezawa $\mathrm{K}$, et al. Is the combination therapy of IKr-channel blocker and left stellate ganglion block effective for intractable ventricular arrhythmia in a cardiopulmonary arrest patient? Cardiol J 2007; 14: $355-$ 365 .

14. Ohashi J, Yasuda S, Miyazaki S, Shimizu W, Morii I, Kurita T, et al. 
Prevention of life-threatening ventricular tachyarrhythmia by a novel and pure class-III agent, nifekalant hydrochloride. J Cardiovasc Pharmacol 2006; 48: 274-279.

15. Tahara Y, Kimura K, Kosuge M, Ebina T, Sumita S, Hibi K, et al Comparison of nifekalant and lidocaine for the treatment of shockrefractory ventricular fibrillation. Circ J 2006; 70: 442-446.

16. Katoh T, Mitamura H, Matsuda N, Takano T, Ogawa S, Kasanuki H. Emergency treatment with nifekalant, a novel class III anti-arrhythmic agent, for life-threatening refractory ventricular tachyarrhythmias: Post-marketing special investigation. Circ J 2005; 69: 1237-1243.

17. Ryan TJ, Antman EM, Brooks NH, Califf RM, Hillis LD, Hiratzka LF, et al. 1999 update: ACC/AHA guidelines for the management of patients with acute myocardial infarction: Executive summary and recommendations. Circulation 1999; 100: 1016-1030.
18. Cheng J, Kamiya K, Kodama I, Toyama J. Heterogeneous distribution of the two components of delayed rectifier $\mathrm{K}+$ current: A potential mechanism of the proarrhythmic effects of methanesulfonanilide class III agents. Cardiovasc Res 1999; 43: 135-147.

19. Kamiya J, Ishii M, Yoshihara K, Oyabe A, Banno H, Katakami T. MS-551: Pharmacological profile of a novel class III antiarrhythmic agent. Drug Dev Res 1993; 30: 37-44.

20. Schwartz PJ, Wolf S. QT interval prolongation as predictor of sudden death in patients with myocardial infarction. Circulation 1978; 57: $1074-1077$

21. Murdock DK, Loeb JM, Euler DE, Randall WC. Electrophysiology of coronary reperfusion: A mechanism for reperfusion arrhythmias. Circulation 1980; 61: 175-182. 